\title{
A comprehensive study of the $\delta$ Scuti star, 57 Tauri (= HR 1351) in the Hyades cluster
}

\author{
M. Paparó ${ }^{1}$, E. Rodriguez ${ }^{2}$, B.J. McNamara ${ }^{3}$, Z. Kolláth ${ }^{1}$, A. Rolland ${ }^{2}$, S.F. Gonzalez-Bedolla ${ }^{4} \dagger$, Jiang Shi-yang ${ }^{5}$, \\ and Li Zhi-ping ${ }^{5}$ \\ 1 Konkoly Observatory, 1525 Budapest XII, P.O. Box 67, Hungary \\ 2 Instituto de Astrofisica de Andalucia, CSIC, Apdo 3004, E-18080 Granada, Spain \\ 3 New Mexico State University, Las Cruces, NM 88003, U.S.A. \\ 4 Observatorio Astronomico Nacional, UNAM, Apdo 877, Ensenada, B.C. 22800, Mexico \\ ${ }^{5}$ Beijing Astronomical Observatory, Chinese Academy of Sciences, Beijing 100080, China
}

Received March 30; accepted August 6, 1999

\begin{abstract}
A comprehensive frequency analysis of 57 Tau, supposed only a static pulsational arrangement, is presented based on 54 nights of photometric data collected between 1981 and 1995 (althogether 232 hours). The presence of individual frequencies is confirmed by intercomparing four subsets of this data set, however, the uncertainties in data coming from the sparse nights and low signal weaken the conclusion on some results. The entire data base is then used to resolve closely spaced peaks. Twelve frequencies are indentified using this process. Further multisite observations are recommended to confirm the present frequency solution. Two periods, with a high probability of being real, are found in the low frequency domain. They are suggested to arise from binarity or $g$ modes, as in $\gamma$ Dor stars. Single unusual cycles, possessing large amplitude, or displaying asymmetry, have been localized. A coherent frequency at $29.83 \mathrm{c} / \mathrm{d}$ with variable amplitude was found. The nonstandard behaviour of the light variation indicates that nonlinear and/or nonstationary processes could be very important in the investigation of $\delta$ Scuti observations.
\end{abstract}

Key words: stars: individual: 57 Tau $=$ HR 1351 - stars: oscillations — stars: variables: $\delta$ Sct — stars: binaries: spectroscopic

\section{Introduction}

The Hyades cluster is the nearest, widely observed open cluster in our Galaxy. It is regarded as a fundamental object in calibrating the cosmic distance scale and in investigating the evolution of the chemical composition in the

Send offprint requests to: M. Paparó
Galaxy. Therefore, the distance and the metal abundance of the Hyades have been widely discussed by many authors (references in Krolikowska 1992).

The Hyades cluster can also be used as a laboratory for the study of the internal structure of stars. The pulsational behaviour (frequency range of excited modes) of a star belonging to a cluster can give severe constraints on pulsation models. Moreover, a comparison of the pulsational behaviour of two pulsating stars in the same cluster (initial chemical composition and age etc. are equal) is an extremely powerful tool for obtaining such constraints.

The idea of investigating of a set of short period, lowamplitude $\delta$ Scuti stars in the Hyades was outlined by Géza Kovács in the late seventies at Konkoly Observatory. A Hyades member, $\theta^{2}$ (78) Tau, is one of the best-studied low-amplitude $\delta$ Scuti stars (Kovács \& Paparó 1989; Breger et al. 1989). 57 Tau was, in principle, a selected target of this project but compact observations were obtained at Konkoly Observatory only from the late eighties onwards.

A frequency analysis of 57 Tau (similar to $\theta^{2}$ (78) Tau) has high astroseismological potential for three reasons: (i) it belongs to the Hyades, (ii) it has been suggested to be a member of a binary system, and (iii) it exhibits multimodal pulsations. Three advantages exist when considering these two stars 1) their membership in the Hyades allows us to define a range of hydrogen and helium abundances, and age, which are fundamental properties for numerical calculations, 2) from the distance modulus to the cluster, the absolute luminosities of these stars can be determined and 3 ) the position of $\theta^{2}$ (78) Tau and 57 Tau on the Hyades colour-magnitude diagram are slightly different; $\theta^{2}(78)$ Tau is close to the turn-off point but 57 Tau is situated closer to the main sequence, and further from the turn-off point of the cluster. 
The light variability of 57 Tau was first discovered by Millis (1967) and confirmed by Horan (1979). Its variability was disputed by Eggen (1970), however, the beats present in the light curve could be responsible for his claim that light from this star is constant. Finally, 57 Tau is regarded as a $\delta$ Scuti type variable, with an amplitude of 0.02 , in the catalogue of $\delta$ Scuti stars (Rodriguez et al. 1994; Lopez de Coca et al. 1990).

A primary analysis of the period ratio of the dominant modes in this star, based on 13 nights of Strömgren $b$ data, has been published by McNamara (1983). The 0.785 period ratio suggests fundamental and first overtone radial pulsation, as is expected for a $\delta$ Scuti star situated near to the red edge of the instability strip. The amplitudes of $\Pi_{0}$ and $\Pi_{1}$ were reported to be exceptionally small being 0.003 and 0.002 , respectively.

The Fourier analysis of two $\delta$ Scuti stars (57 Tau and $58 \mathrm{Tau}$ ) in the Hyades cluster is reported by $\mathrm{Fu}$ Jian-ning et al. (1996). Two frequencies (18.221 and $20.438 \mathrm{c} / \mathrm{d}$ ) were obtained with similar small amplitudes (McNamara 1983). However, the period ratio of the new analyses did not find a period ratio within the range expected for a radial pulsator.

The aim of this paper is to perform an overall analysis for all the available observation of 57 Tau. However, the quality of data, including their sparse distribution and the low signal, allowed only the investigation of a static pulsational arrangement supposed no changes in amplitudes and phases and ruled out any kind of investigation of resonance effects. If we see a search for amplitude variability as an important scientific goal, we could not achieve that level in our comprehensive analysis. Unpublished and new observations are discussed in Sect. 2. The mean light level variation is investigated in Sect. 3. The frequency analysis is considered in Sect. 4 . The state of our present knowledge on 57 Tau is discussed in Sect. 5 .

\section{Observations}

Table 1 lists all the available observation dates included in our analysis. Data collection on 57 Tau are spread over more than a decade. Besides a few hours of early observations, in which, the light variations were discovered, the first extended observations of 57 Tau were obtained by B.J. McNamara. This data set consists of 16 nights from 1981-83 and yielded 934 differential Strömgren $b$ photometric measurements (68.9 hours) using the NMSU 16 inch telescope.

Apart from the single night in 1984, 57 Tau has been regularly observed, by M. Paparó, from the Konkoly Observatory in the 1986/87 season, and Johnson $V$ colour data were obtained over a 38.9 hour during 9 nights.

Two coordinated observing campaigns were organized by M. Paparó in 1989, including Chinese and Hungarian observatories, and in 1995 which included
Table 1. The journal of observations. Four compact data sets exist

\begin{tabular}{|c|c|c|c|c|c|}
\hline Date & $\begin{array}{l}\text { HJD- } \\
2400000\end{array}$ & $\begin{array}{l}\text { length } \\
\text { (hours) }\end{array}$ & $N$ & filter & Obs. \\
\hline Oct. 25/26 1981 & 44903 & 5.4 & 87 & $b$ & BJM \\
\hline Oct. 26/27 1981 & 44904 & 4.5 & 64 & $b$ & BJM \\
\hline Nov. 1/2 1981 & 44910 & 4.1 & 60 & $b$ & BJM \\
\hline Nov. 4/5 1981 & 44913 & 4.3 & 52 & $b$ & BJM \\
\hline Nov. 9/10 1981 & 44918 & 5.7 & 69 & $b$ & BJM \\
\hline Nov. 23/24 1981 & 44932 & 5.1 & 60 & $b$ & BJM \\
\hline Nov. 24/25 1981 & 44933 & 5.2 & 65 & $b$ & BJM \\
\hline Nov.30/Dec1 1981 & 44939 & 1.1 & 12 & $b$ & BJM \\
\hline Dec. 7/8 1981 & 44946 & 5.4 & 70 & $b$ & BJM \\
\hline Dec. 28/29 1981 & 44967 & 4.9 & 59 & $b$ & BJM \\
\hline Jan. 25/26 1982 & 44995 & 3.7 & 46 & $b$ & BJM \\
\hline Jan. 26/27 1982 & 44996 & 3.5 & 44 & $b$ & BJM \\
\hline Feb. 15/16 1982 & 45016 & 2.7 & 34 & $b$ & BJM \\
\hline Feb. 20/21 1982 & 45021 & 2.6 & 40 & $b$ & BJM \\
\hline Jan. 3/4 1983 & 45338 & 5.6 & 83 & $b$ & BJM \\
\hline Jan. 4/5 1983 & 45339 & 5.1 & 89 & $b$ & BJM \\
\hline Dec. 14/15 1984 & 46049 & 4.3 & 86 & V & MP \\
\hline Sep. 19/20 1986 & 46693 & 3.1 & 70 & V & MP \\
\hline Sep. 20/21 1986 & 46694 & 1.6 & 29 & $V$ & MP \\
\hline Oct. 18/18 1986 & 46722 & 3.6 & 68 & $V$ & MP \\
\hline Nov. 28/29 1986 & 46763 & 6.0 & 106 & V & MP \\
\hline Nov. 29/30 1986 & 46764 & 4.7 & 90 & $V$ & MP \\
\hline Nov. 30/Dec1 1986 & 46765 & 2.5 & 50 & $V$ & MP \\
\hline Dec. 3/4 1986 & 46768 & 3.3 & 62 & $V$ & MP \\
\hline Jan. 20/21 1987 & 46816 & 5.1 & 89 & $V$ & MP \\
\hline Feb. 5/6 1987 & 46832 & 4.7 & 84 & $V$ & MP \\
\hline Jan. 13/14 1989 & 47540 & 5.1 & 28 & $V$ & JSy, LZp \\
\hline Jan. 14/15 1989 & 47540.9 & 5.9 & 39 & V & JSy, LZp \\
\hline Jan. 14/15 1989 & 47541.2 & 2.9 & 22 & V & $\mathrm{MP}$ \\
\hline Jan. 15/16 1989 & 47541.9 & 6.8 & 59 & $V$ & JSy, LZp \\
\hline Jan. 16/17 1989 & 47542 & 5.4 & 43 & V & JSy, LZp \\
\hline Jan. 17/18 1989 & 47543 & 6.6 & 29 & $V$ & JSy, LZp \\
\hline Jan. 18/19 1989 & 47544.9 & 3.6 & 33 & $V$ & JSy, LZp \\
\hline Jan. 18/19 1989 & 47545.3 & 2.5 & 22 & V & MP \\
\hline Jan. 19/20 1989 & 47546.0 & 4.6 & 31 & $V$ & JSy, LZp \\
\hline Jan. 20/21 1989 & 47546.9 & 6.6 & 60 & V & JSy, LZp \\
\hline Jan. 24/25 1989 & 47551 & 2.7 & 24 & $V$ & MP \\
\hline Jan. 25/26 1989 & 47552 & 4.2 & 41 & V & MP \\
\hline Dec. 7/8 1989 & 47868 & 6.0 & 79 & $V$ & $\mathrm{ZK}$ \\
\hline Dec. 8/9 1989 & 47869 & 7.2 & 117 & $V$ & $\mathrm{ZK}$ \\
\hline Oct. 31995 & 49994 & 4.4 & 94 & uvby & $\mathrm{ER}, \mathrm{MP}$ \\
\hline Oct. 51995 & 49996 & 4.2 & 75 & uvby & $\mathrm{ER}, \mathrm{MP}$ \\
\hline Oct. 61995 & 49997 & 5.4 & 73 & uvby & ER, MP \\
\hline Oct. 71995 & 49998.4 & 1.5 & 23 & uvby & $\mathrm{ER}, \mathrm{MP}$ \\
\hline Oct. 81995 & 49999.4 & 5.9 & 100 & uvby & $\mathrm{ER}, \mathrm{MP}$ \\
\hline Oct. 81995 & 49999.9 & 2.9 & 41 & uvby & GB \\
\hline Oct. 91995 & 50000.4 & 5.0 & 85 & uvby & ER, MP \\
\hline Oct. 91995 & 50000.8 & 2.6 & 25 & uvby & GB \\
\hline Oct. 101995 & 50001 & 5.2 & 178 & uvby & $\mathrm{ER}, \mathrm{MP}$ \\
\hline Oct. 121995 & 50003 & 1.4 & 21 & uvby & GB \\
\hline Oct. 131995 & 50004 & 2.4 & 32 & uvby & GB \\
\hline Oct. 141995 & 50005 & 5.2 & 84 & uvby & $\mathrm{AR}$ \\
\hline Oct. 151995 & 50006 & 4.9 & 79 & uvby & $\mathrm{AR}$ \\
\hline Oct. 161995 & 50007 & 3.3 & 38 & uvby & GB \\
\hline 54 nights & & 232.2 & 3243 & & \\
\hline
\end{tabular}


Spanish, Mexican, Chinese and Hungarian observers. Unfortunately weather conditions in China and Hungary were poor in 1995, so no usable data were obtained.

The Chinese observations were obtained at the Xinglong Station of Beijing Astronomical Observatory on the $60 \mathrm{~cm}$ reflector with a classical single channel photoelectric photometer through a Johnson $V$ filter. Over 8 nights, 45 hours of observations were collected by Jiang Shi-yang and Li Zhi-ping.

As a consequence of bad weather, only 4 nights (12.9 hours) were obtained by M. Paparó during the coordinated campaign in early 1989.

Two additional nights (13.1 hours) were obtained by Z. Kolláth at Konkoly Observatory at the end of 1989. All the Hungarian observations were obtained on the $0.5 \mathrm{~m}$ reflector of Piszkéstetö, the mountain station of Konkoly Observatory, through a $V$ filter close to the Johnson $U B V$ system. A single channel integrating photometer, developed and build by Géza Virághalmy, the technical deputy director of Konkoly Observatory, was equipped with an EMI 9502S photomultiplier. The photometer is characterized by high instrumental zero-point stability.

In 1995, 7 nights of observations (31.6 hours) were collected in Spain by E. Rodriguez and M. Paparó and 2 nights (10.1 hours) by A. Rolland. The $0.90 \mathrm{~m}$ telescope at Sierra Nevada, with the six-channel Danish photometer, was used for obtaining Strömgren uvby colours.

Five nights of observations (12.5 hours) were obtained by S.F. Gonzalez-Bedolla on the $1.5 \mathrm{~m}$ telescope at San Pedro Martir in Mexico. Simultaneous Strömgren uvby data were collected with a Danish photometer.

Altogether, 232 hours of data obtained on 54 nights at different sites are included in our comprehensive analysis on 57 Tau. All observations were obtained as differential measurements, so variable, comparison and sky background were measured alternately. Except for the multi-site campaign in 1995, the only comparison star generally used was HR 1358 (= HD 27483). The analysis of the errors and/or noise effects and/or instrumental effects could not be done for most part of the data set as in the case of two comparison stars. In the course of the multisite campaign in 1995 a second comparison star, HR 1430 (= HD 28556), was also observed, to check the constancy of the primary comparison star.

Reductions were independently made by the observer for each data set. In principle, this could introduce instrumentation effect in the whole set, especially at low frequencies. The standard method of differential extinction corrections was applied. In most cases, nightly extinction coefficients were used, in other cases, mean values of the extinction coefficients for a given site were accepted.

The accuracy of the observations are not homogeneous, specifically the data collected in the coordinated 1989 campaign from China and Hungary have larger scatter. To compensate for this, sequences of three observations were combined for the Chinese data compared to

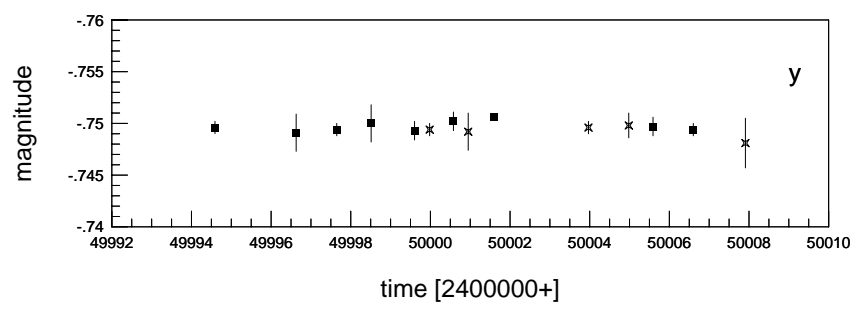

Fig. 1. The nightly mean values of HR $1430-$ HR 1358, the two comparison stars of 57 Tau in 1995. $3 \sigma$ error bars are given

the light curves published by $\mathrm{Fu}$ et al. (1996). A similar process involving the averaging of 2 points was used for the Hungarian data obtained at the beginning of 1989. The impression that the light curves consist of poor quality data, which comes from the low signal/noise value, is a consequence of the low amplitude light variability of 57 Tau. The accuracy of a single measurement, given below, is similar to that usually obtained for $\delta$ Scuti-type stars.

\section{Mean light level}

In previous analyses of 57 Tau (e.g., Fu Jian-ning et al. 1996), differences in the nightly mean magnitude of $57 \mathrm{Tau}$, relative to the comparison star, have been mentioned, but not discussed in depth. After applying the nightly determined extinction coefficient, a nightly zero-point adjustment was made, which was thought to be caused by unknown observational problems. In our analysis we were interested in the thorough study of the low frequency domain of the spectrum, since all the available data sets displayed regular features in this range (Paparó et al. 1999).

Since the variability of the comparison star and the incorrect transformation of the instrumental systems may effect our conclusions for the mean light level of 57 Tau, both the constancy of the comparison stars, and the effect of data's homogenizations were carefully investigated.

\subsection{Comparison stars}

The constancy check of the comparison star, HR 1358, is based on the simultaneous observation of HR 1358 and HR 1430 in the course of the multi-site campaign on 57 Tau in 1995 in Spain and Mexico. Over 14 nights, 54.35 hours, 543 Strömgren uvby measurements were collected. The nightly average values versus the mean time of the given observing run are shown in Fig. 1. The Spanish data are marked by filled squares and the Mexican ones by crosses.

The average Strömgren values of the differential magnitudes for HR 1430 - HR 1358 for all data, from the Spanish and Mexican data sets, are listed in Table 2. 
Table 2. Mean values of HR 1430 - HR 1358

\begin{tabular}{llllll}
\hline Data & $N$ & $u$ & $v$ & $b$ & $y$ \\
\hline All & 543 & $-0.7726 \pm .0002$ & $-1.0153 \pm .0001$ & $-0.8948 \pm .0001$ & $-0.7496 \pm .0001$ \\
Spanish & 473 & $-0.7725 \pm .0002$ & $-1.0154 \pm .0001$ & $-0.8948 \pm .0001$ & $-0.7496 \pm .0001$ \\
Mexican & 70 & $-0.7733 \pm .0008$ & $-1.0151 \pm .0004$ & $0.8950 \pm .0003$ & $-0.7492 \pm .0004$ \\
Catalogue & & -.764 & -1.025 & -0.908 & -0.768 \\
\hline
\end{tabular}

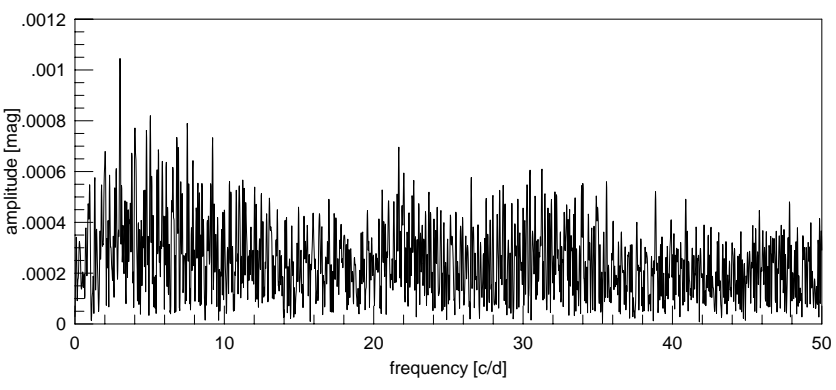

Fig. 2. Amplitude spectrum of HR 1430 - HR 1358, the two comparison stars of 57 Tau in y colour. The peak at $3.02 \mathrm{c} / \mathrm{d}$ is possibly resulted from the errors in extinction correction

The $u b v y$ values taken from Mermilliod et al. (1997) are given at the bottom of the table.

The mean values are given with high accuracy. The standard deviation of the measurements around the mean, $\approx 0 .{ }^{\mathrm{m}} 003$ in $v b y$ colours and $\approx 0.005$ in $u$ colour, could be regarded as the error of observations in 1995. The Spanish and Mexican mean values do not greatly differ which means that the two instrumental photometric systems are very close to each other. This is not surprising since the detector attached to both telescopes is the Danish photometer. The difference, concerning the catalogue values, is probably due to the difference of the instrumental and standard Strömgren systems.

While the low values of errors for the comparison stars suggest constancy of both comparison stars, a frequency analysis of HR 1430 - HR 1358 differences was carried out in a range of $0-50$ cycles per day. The amplitude spectrum in $y$ colour is given in Fig. 2. The amplitude spectra $(A(f))$ are characterized by its mean values $\langle A(f)>$ i.e. $0.408 \pm 0.213,0.243 \pm 0.135,0.216 \pm 0.119$ and $0.255 \pm$ $0.137 \mathrm{mmag}$ in the uvby colours, respectively. Although for the most part, the amplitude spectrum does not show regular structure, a peak might be present in vby at 3.028, 3.025 and $3.022 \mathrm{c} / \mathrm{d}$ frequency with an amplitude of 0.90 , 0.72 and $0.98 \mathrm{mmag}$, respectively.

The spectral window pattern, due to the multi-site observation, is not seen around this peak. The significance limit, 4 times the mean value of the residual spectrum (Breger et al. 1993), has been accepted, and is 0.94, 0.83 and $0.96 \mathrm{mmag}$ in $v b y$, respectively. The distinct peak is only above the significance limit for the $y$ colour.

Could this periodicity be caused by a difference in the mean light level between the two sites separated by 8 hours

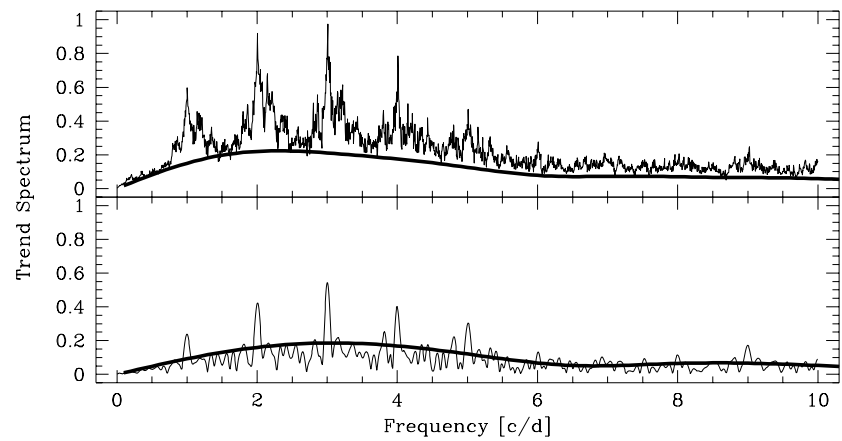

Fig. 3. The effect of incorrect extinction coefficient. Upper panel for the whole data set, lower panel for the 1995 data. Uncorrelated trend-spectra (UTS, thick curve), systematic trend-spectra (STS, thin curve)

in longitude? As Table 2 and Fig. 1 show, the mean values of HR 1430 - HR 1358 are the same for both observatories with slightly larger scatter for the Mexican observations caused by the shortness of the data set. If the periodicity at $3.02 \mathrm{c} / \mathrm{d}$ is caused by some special instrumental circumstances at the different sites, the separate data would not show the periodicity. A periodicity at $3.026 \mathrm{c} / \mathrm{d}$ (with an amplitude of $0.96 \mathrm{mmag}$ ) with a spectral window pattern around it, has been found for the Spanish data. According to the significance test, the amplitude of the period is below the significance level (1.04 mmag). The Mexican data are too short for separately checking the period.

Instrumental magnitudes are often contaminated by small nightly trends, e.g., due to the errors in the extinction coefficients. To estimate the possible spectral distribution of this noise, trend-spectra were introduced by Kolláth \& Paparó (1999). These spectra are given for two extreme cases: for uncorrelated trends and for systematic trends. In the first case the mean spectrum behaves like a coloured noise (continuous spectrum), while for systematic errors the peaks from the spectral window appear also in the trend spectrum. In general the spectral noise generated by the trends are between these two specific cases. We have to note that the trend spectra give no information on the amplitude of the noise due to the trends. Like the spectral window it gives the normalized spectral distribution only.

In Fig. 3. both the uncorrelated trend-spectra (UTS, thick curve) and the systematic trend-spectra (STS, thin curve) are displayed for the whole data set and also for 
Table 3. Mean values of 57 Tau - HR 1358 for different sites

\begin{tabular}{lll}
\hline Sites & $N$ & mean values \\
\hline Hungarian Johnson $V$ & 1039 & -0.6081 \\
Chinese Johnson $V$ & 322 & -0.57106 \\
Spanish + Mexican Strömgren $y$ & 948 & -0.57460 \\
Spanish + Mexican Strömgren $b$ & 948 & -0.70195 \\
McNamara Strömgren $b$ & 934 & -0.79442 \\
\hline
\end{tabular}

the 1995 data. Both panels show that the effect of errors in the extinction coefficients is most pronounced around $3 \mathrm{c} / \mathrm{d}$ frequency values. A similar conclusion has been obtained by Poretti \& Zerbi (1993) and Breger \& Beichbuchner (1996) The $3.02 \mathrm{c} / \mathrm{d}$ frequency in the comparison light-curve is therefore probably related to errors in extinction correction.

We conclude that the differential light curve of the two comparison stars, HR 1430 - HR 1358, does not show any evidence for variability, although it is slightly effected by extremely small errors in the atmospheric extinction coefficient. Any periodicity in the differential light curve of 57 Tau and HR 1358 should be attributed to 57 Tau as intrinsic variability.

\subsection{Homogenization of data}

Combining all of the 57 Tau data is not a trivial task. We have the advantage of similar spectral type comparison stars, so the colour dependence of instrumental systems can be neglected. In the milli-magnitude range, however, different photometer/filter combinations lead to different zero-points, even if the same comparison stars are used. This fact needs to be considered when different data sets from different locations are combined. A complicating factor is that, not only are Johnson $V$ \& Strömgren $y$ data involved in our analyses, but Strömgren $b$ colour data were also included.

In order to combine the data from the different sites adjustments were made. For each observatory the photometric zero-point of 57 Tau relative to HR 1358 was determined by averaging all the the data from each observatory. The mean values obtained are listed in Table 3 .

In order to get the highest possible frequency resolution, we treated all the observational data together, regardless of the filters used for the measurements. All the available data (even B.J. McNamara's Strömgren $b$ colour data) are shifted to the mean value of the Spanish + Mexican Strömgren $y$ colour. Shifts of $+0{ }^{\mathrm{m}} 0335$, -0 . 00354 and +0.21982 were applied for the Hungarian \& Chinese Johnson $V$, and B.J. McNamara's Strömgren $b$ data. Johnson $V$ \& Strömgren $y$ and $b$ measurements were regarded to be identical except for the different zero-points.

The largest error introduced in our Fourier spectra is due to the mixing of the early $b$ data with the later $y$ or

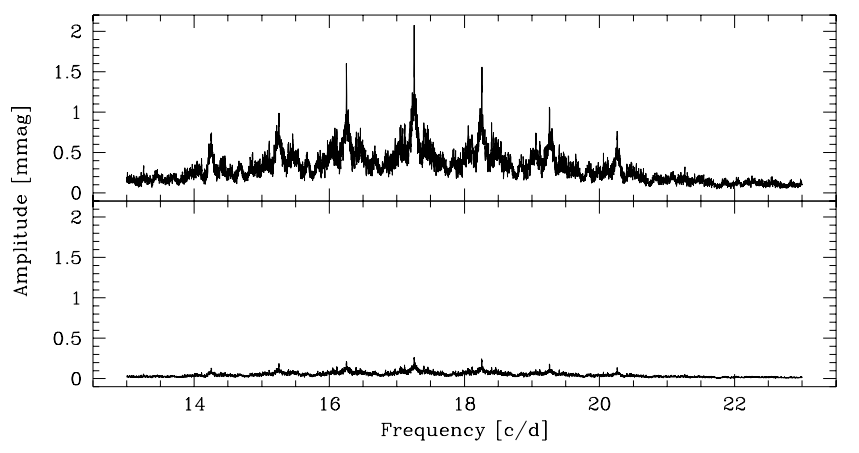

Fig. 4. The effect of variable amplitude and phase shift for the frequency analyses. The amplitude of the signal was increased by 40 percent and the signal was shifted in phase by $10^{\circ}$ for the times of $b$ observations. The spectral residual is no more than $0.03 \mathrm{mmag}$ for the highest amplitude component

$V$ observations. We have estimated this spectral noise by the following experiment: We made a synthetic signal with a single frequency with the highest amplitude $(2 \mathrm{mmag})$ from the real data, but we increased the amplitude of the signal by 40 percent and shifted the phase by $10^{\circ}$ for the times of $b$ observations (overestimated the generally obtained amplitude ratio and phase differences between the $y$ and $b$ colours). Then we treated these data like the real observations: we prewhitened the synthetic light-curve with the average amplitude (obtained by least-square fitting). The spectra of the test signal and the prewhitened data are displayed in Fig. 4. From this test it can be concluded that even with a 40 percent difference in the amplitudes and $10^{\circ}$ difference in phases the spectral residual is no more than $0.3 \mathrm{mmag}$ for the highest amplitude component. More complicated cases (more frequencies, even not all excited simultaneously) are going to be discussed by Kolláth \& Paparó (1999).

Our test, in this ideal case, proved that we can extend the time-base of the analysis for the B.J. McNamara's observations obtained in the early eighties for the Strömgren $b$ colour for finding the periodicities in the star.

In the final step, B.J. McNamara's Strömgren $b$ observations were shifted to the Spanish \& Mexican mean value of Strömgren $b$ colour for determining the amplitude of the pulsation frequencies in $b$ colour. The shift (0. 09247) between the two Strömgren systems is much larger than expected for well-defined Strömgren systems. No trivial explanation exists but different photomultiplier tubes were employed at these sites and much of the difference is thought to arise from this fact.

As a result of this process, the low-frequency part of the amplitude spectrum is cleaned and any dominant periodicity in this range is unlikely to be caused by the inhomogeneity of different data sets. 
Table 4. Frequency solutions for the subsets of data

\begin{tabular}{|c|c|c|c|c|c|c|c|c|c|c|c|c|c|}
\hline \multirow{3}{*}{ Subset } & \multirow{3}{*}{$\begin{array}{l}\text { Nights } \\
N\end{array}$} & \multicolumn{12}{|c|}{ Frequency in c/d, Amplitude in mmag } \\
\hline & & $F_{1}$ & $F_{2}$ & $F_{3}$ & $F_{4}$ & $F_{5}$ & $F_{6}$ & $F_{7}$ & $F_{8}$ & $F_{9}$ & $F_{10}$ & $F_{11}$ & $F_{12}$ \\
\hline & & $A_{1}$ & $A_{2}$ & $A_{3}$ & $A_{4}$ & $A_{5}$ & $A_{6}$ & $A_{7}$ & $A_{8}$ & $A_{9}$ & $A_{10}$ & $A_{11}$ & $A_{12}$ \\
\hline \multirow[t]{2}{*}{$81 / 82$} & 14 & $0.654^{*}$ & $0.804^{*}$ & 1.021 & $7.232^{*}$ & 14.762 & 16.594 & 19.048 & $18.260^{*}$ & - & $20.406^{*}$ & 25.556 & $29.837^{*}$ \\
\hline & 762 & 2.13 & 2.80 & 1.14 & 1.90 & 2.07 & 1.60 & 2.18 & 1.06 & - & 2.40 & 1.68 & 1.88 \\
\hline \multirow[t]{2}{*}{$86 / 87$} & 9 & $0.659^{*}$ & $0.845^{*}$ & - & 6.262 & - & 16.626 & $18.202^{*}$ & - & - & 20.411 & $25.843^{*}$ & - \\
\hline & 648 & 0.96 & 1.12 & - & 1.40 & - & 1.78 & 3.40 & - & - & 1.23 & 1.77 & - \\
\hline \multirow[t]{2}{*}{89} & 14 & $0.626^{*}$ & $0.802^{*}$ & 1.119 & 8.002 & - & 16.493 & - & $18.247^{*}$ & - & $21.434^{*}$ & - & 29.527 \\
\hline & 627 & 1.25 & 1.89 & 1.93 & 1.26 & - & 1.58 & - & 3.76 & - & 2.21 & - & 1.24 \\
\hline \multirow[t]{2}{*}{95} & 14 & $0.665^{*}$ & $0.813^{*}$ & - & 7.237 & 14.173 & 16.214 & - & $18.230^{*}$ & - & $20.446^{*}$ & - & $29.843^{*}$ \\
\hline & 948 & 0.89 & 2.51 & - & 0.94 & 0.68 & 2.50 & - & 2.73 & - & 0.96 & - & 1.35 \\
\hline \multirow[t]{2}{*}{ Hung. } & 16 & $0.657^{*}$ & $0.831^{*}$ & - & 7.094 & 14.597 & 16.232 & $18.201^{*}$ & - & - & 20.429 & 24.800 & 29.866 \\
\hline & 1039 & 1.20 & 2.00 & - & 1.41 & 1.25 & 1.66 & 2.61 & - & - & 1.53 & 1.75 & 1.19 \\
\hline Chin. & 9 & 1.628 & 0.785 & $1.131^{*}$ & 6.596 & - & 16.489 & - & $18.235^{*}$ & - & $20.438^{*}$ & 25.758 & - \\
\hline \multirow[t]{2}{*}{$81-87$} & 26 & $0.657^{*}$ & $0.803^{*}$ & 1.162 & 7.093 & - & - & $18.011^{*}$ & $18.258^{*}$ & $18.403^{*}$ & $20.416^{*}$ & 25.841 & 29.836 \\
\hline & 1668 & 1.90 & 1.79 & 1.38 & 1.21 & - & - & 2.11 & 1.32 & 1.36 & 1.37 & 1.36 & 1.23 \\
\hline \multirow[t]{2}{*}{ 86-89 } & 23 & - & $0.803^{*}$ & 1.118 & - & - & 16.232 & $18.201^{*}$ & - & - & 20.413 & 26.673 & - \\
\hline & 1275 & - & 2.04 & 1.64 & - & - & 1.55 & 2.60 & - & - & 1.84 & 1.26 & - \\
\hline \multirow[t]{2}{*}{$81-89$} & 40 & $0.660^{*}$ & $0.793^{*}$ & 1.122 & $7.019^{*}$ & - & 17.615 & $18.216^{*}$ & $18.260^{*}$ & - & $20.441^{*}$ & $25.553^{*}$ & $29.836^{*}$ \\
\hline & 2295 & 1.89 & 1.40 & 1.61 & 1.00 & - & 1.49 & 1.60 & 1.57 & - & 1.62 & 1.31 & 1.31 \\
\hline \multirow[t]{2}{*}{$81-95$} & 54 & $0.657^{*}$ & $0.803^{*}$ & $1.119^{*}$ & $7.223^{*}$ & 14.161 & 16.738 & $18.220^{*}$ & $17.257^{*}$ & 20.218 & $20.441^{*}$ & $24.555^{*}$ & $29.839^{*}$ \\
\hline & 3243 & 1.26 & 1.80 & 1.07 & 1.00 & 0.95 & 1.06 & 1.41 & 2.07 & 1.35 & 1.24 & 1.13 & 1.29 \\
\hline \multirow[t]{2}{*}{$81 \& 95 \mathrm{~b}$} & 30 & $0.657^{*}$ & $0.805^{*}$ & - & $7.233^{*}$ & 15.189 & 16.739 & - & $17.248^{*}$ & 18.472 & $20.406^{*}$ & $24.555^{*}$ & $29.838^{*}$ \\
\hline & 1882 & 1.21 & 3.01 & - & 1.38 & 1.60 & 0.93 & - & 2.11 & 1.27 & 1.68 & 1.56 & 1.51 \\
\hline
\end{tabular}

\subsection{Mean light level of 57 Tau}

The nightly mean photometric value (in a given filter) of 57 Tau, relative to the comparison star, HR 1358, versus the mean time of an observing run for each site was checked for periodicity. The method of averaging is useful if the periodicity is larger than an observing run, and seems to be stable if the pulsational periods are remarkably shorter, many cycles are averaged in a run.

A simple view of the mean light level does not show a cyclic pattern. After homogenization, the Strömgren $y \&$ $b$ mean values of $57 \mathrm{Tau}-\mathrm{HR} 1358$ are $-0.5744 \pm 0.0019$ and $-0.7020 \pm 0.0027$, respectively. The low value of the scatter for the mean light level suggests that if there is any variability, it has a low amplitude or periodicity near to $1 \mathrm{c} / \mathrm{d}$.

A frequency analysis of the mean light values was carried out in a range of $0-4$ cycles/day. A peak of highest amplitude at $0.80866(0.00163)$ and $0.80231(0.00318)$ cycles/day was found in $y \& b$, respectively. According to a significance test, the periodicity is significant.

Although the amplitude of mean light level variation seems to be extremely low, it is comparable to the amplitude of the pulsation modes of 57 Tau, as shown in Fig. 5 .

The final frequencies connected to the mean light level variation of 57 Tau were obtained as part of the final frequency solution given in the next paragraph.

\section{Analysis}

A comprehensive analysis of a pulsating variable star is based on the assumption that the star's pulsation behaviour is determined by the inner structure of the stars, which most of the time does not change dramatically over a few years or a decade or so. Evolutionary period changes or instability in the amplitudes should be expected according to the evolutionary and pulsation theory but the stationarity of the pulsation as an initial assumption could be accepted.

If we accept the idea mentioned above, we could see our comprehensive analysis as a set of data where the same physical process is sampled at different times and sites (which means different longitude in our case) with different data distribution involving two runs of coordinated observation. The distinct subsets of data could be regarded as a physical process convolved with different window pattern, which produce different distribution of side lobes around the real frequencies. Although none of the subsets are long enough to get a well-established solution for the real physical process going on in the star, the peaks which appear in each subset suggest a high probability of a peak being a real frequency.

However, we should emphasize that in a comprehensive analysis some of the frequencies and specially their amplitudes can be regarded to be on a level of a guess not a fact. In the case of data sets like this a search for 

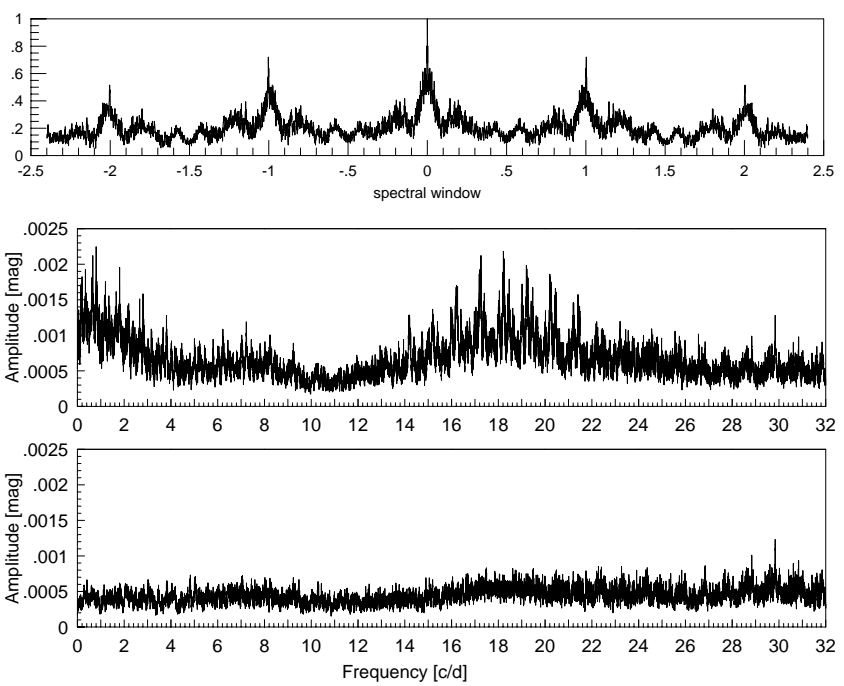

Fig. 5. The spectral window, the unprewhitened and residual amplitude spectrum of 57 Tau for the whole data set (198195). The amplitudes in the low frequency part are comparable to the amplitude of pulsation modes in the $p$-mode region. The low frequency part of the residual spectrum seems to be completely clean. The residual spectrum has a significant peak at $29.8386 \mathrm{c} / \mathrm{d}$ with large amplitude fluctuations

amplitude variability is hopeless. No phases and errors are given in our paper neither for the frequency solution for the subsets nor for the finally accepted frequency solution because it would suggest that the presented mathematical description of the light variation of 57 Tau is ready for theoretical modelling.

The multi-frequency analysis of 57 Tau was performed with the MUFRAN programme (Kolláth 1990). MUFRAN (MUlti FRrequency ANalysis) is a collection of methods for period determination, sine fitting for observational data and graphics display routines. At each step, all previous frequencies were computed and successive prewhitening was avoided.

\subsection{Subsets}

As a first step, the different data sets were analysed separately. Of course the shorter time period covered by these independent data sets does not allow the precise value of the frequencies to be determined or closely spaced frequencies to be resolved. We are not going to discuss the frequency analyses of subsets in detail, but the frequencies detected from the independent subsets or different combinations of data sets, with their amplitudes, are summarized in Table 4. Those frequencies, which are identified also in the unprewhitened spectrum of the subsets and maybe more free of bias than the others, are marked by asterisk.

We should include some general remarks. First, it is very encouraging that for different data sets, and spectral
Table 5. Finally accepted frequency solution for 57 Tau. * Marks the frequencies that can be localized in the unprewhitened spectrum

\begin{tabular}{lll}
\hline $\begin{array}{l}\text { Frequencies } \\
\mathrm{c} / \mathrm{d}\end{array}$ & $\begin{array}{l}\text { Ampl. in } V \\
\mathrm{mmag}\end{array}$ & $\begin{array}{l}\text { Ampl. in } B \\
\mathrm{mmag}\end{array}$ \\
\hline$f_{1} 0.16208$ & 1.27 & 1.27 \\
$f_{2} 0.65745^{*}$ & 1.26 & 1.20 \\
$f_{3} 0.80250^{*}$ & 1.80 & 2.33 \\
$f_{4} 1.11933$ & 1.07 & 0.83 \\
$f_{5} 7.22323$ & 1.00 & 1.23 \\
$f_{6} 14.16131$ & 0.95 & 1.08 \\
$f_{7} 16.73835$ & 1.06 & 1.07 \\
$f_{8} 17.25689^{*}$ & 2.07 & 2.24 \\
$f_{9} 18.21986^{*}$ & 1.41 & 1.32 \\
$f_{10} 20.21810$ & 1.35 & 1.27 \\
$f_{11} 20.44054^{*}$ & 1.24 & 1.51 \\
$f_{12} 24.55519^{*}$ & 1.41 & 1.62 \\
\hline
\end{tabular}

window patterns, several similar frequencies are found. As a consequence of the shortness of the independent data sets, the frequencies are not properly resolved around 18.2 and $20.4 \mathrm{c} / \mathrm{d}$. Depending on the data set one or two dominant peaks are seen in this frequency range but the dominant ones are not always the same. Several frequencies are common to each sample, although in some cases, it is hard to decide between values that differ by $\pm(1-2) \mathrm{c} / \mathrm{d}$. This is a typical result for the discontinuous data sets. However, some guidelines exist that can help to distinguish between the aliases. It is obvious that not all of the peaks situated around $18 \mathrm{c} / \mathrm{d}$ are real frequencies, because there are amplitude variations in the observed light curve from cycle to cycle. Such variations could be better described by beat of frequencies further away from each other.

\subsection{The whole data set}

As a last step of the analysis, all the available data from 1981-1995 were analysed together. We should emphasize that the solutions are obtained from properly sampled spectra. Regarding the longest time base, the spectra were calculated for each $4 \mathrm{c} / \mathrm{d}$ wide range separately, with 90001 points. Figure 5 displays the spectral window, the original and residual amplitude spectra of 57 Tau.

For easier graphical representation we show compressed spectra, i.e. only the maximum values are plotted in each $0.0022 \mathrm{~d}^{-1}$ wide bins of the spectra. Contrary to undersampled spectra the frequencies and amplitudes of the peaks are undisturbed after compression.

The best mathematical representation of the pulsation spectrum of 57 Tau for the entire (1981-95) time-base, supposed a static pulsational arrangement, is given in Table 5 . Amplitudes are given for both $y \& b$ colours. The residual spectrum in Fig. 5. is obtained after prewhitening with the finally accepted frequency solution. 


\subsubsection{Low frequency domain}

In principle, low frequency terms can be an artifact introduced by small misalignements between different subsets (instrumental effects). Such kind of low frequency terms can be hardly cleaned by limited number of frequencies. For 57 Tau four frequencies were accepted in the low frequency domain. The two frequencies at 0.8025 and $0.6575 \mathrm{c} / \mathrm{d}$ are the most certain. Although the difference between the two frequencies is near to a value of an alias peak in the spectral window, the two frequencies can be independently found in the spectrum. After prewhitening with these two, another frequency at $0.805 \mathrm{c} / \mathrm{d}$ appeared but a prewhitening with a frequency at $0.162 \mathrm{c} / \mathrm{d}$ resulted a better residual spectrum. This frequency is not involved in Table 4 since it has not appeared in most of the subsets. The frequency at $1.119 \mathrm{c} / \mathrm{d}$, similar to that obtained from the Chinese data set (see Table 4), appeared only in the last stage of the analysis. The steps of prewhitening can be found in Paparó et al. (1999). The low frequency part of the residual spectrum in Fig. 5 seems to be completely clean. After prewhitening with all frequencies a peak at $3.02141 \mathrm{c} / \mathrm{d}$ with $0.69 \mathrm{mmag}$ amplitude appeared. The fact that the same periodicity appeared in the analyses of the comparison stars, as a result of small errors in the determination of atmospheric extinction, confirms that the previous prewhitening process removed real power.

\subsection{2. $p$-mode region}

Two or three frequencies seem to be excited around 17.2 or 18.2 and one or two around 20.4, although in most subsets only two of these frequencies can be localized in the unprewhitened spectrum. The frequenices accepted in the final solution are the result of many trials but should not be regarded as definite, as solutions in the case of the best-studied $\delta$ Scuti stars (even the integer part can be ambiguous). The frequencies at 7.2 and $24.5 \mathrm{c} / \mathrm{d}$ do not appear to be disturbed by other frequencies but the proper cycle counting $(+1 \mathrm{c} / \mathrm{d}$ alias $)$ is questionable. The frequencies at 14.1 and $16.7 \mathrm{c} / \mathrm{d}$ may be influenced by the previous steps of prewhitening.

Although the amplitudes of frequencies are extremely low, all of them past the significance test discussed earlier. The residual spectrum in the $p$-mode region is not completely cleaned. The spectrum shown in Fig. 5 (bottom panel) has a significant peak at $f_{13}=29.8386 \mathrm{c} / \mathrm{d}$, however an inspection of the fit indicates large amplitude fluctuations of this mode. Because of the small signal to noise ratio, it is impossible to obtain time-dependent amplitudes for this oscillation. From amplitude fitting we could not even determine whether this mode exists permanently in the data. However the phase of the oscillation can give valuable information.

To check whether this oscillation is present coherently in the whole data set, we calculated the Fourier phase di-

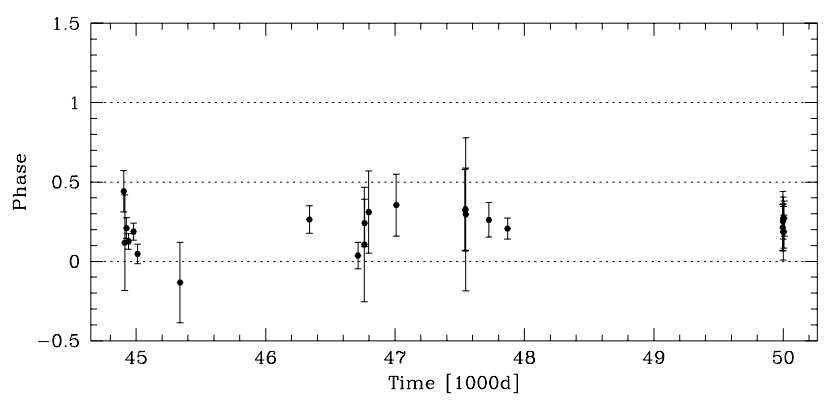

Fig. 6. The Fourier-phase diagram for the frequency at $29.83 \mathrm{c} / \mathrm{d}$ value. The well-defined phase value over the entire dataset represent the coherent behaviour of the periodicity as mentioned in the text

agram (Paparó et al. 1998) for this frequency (Fig. 6). To avoid errors due to other periodicities we first prewhitened the data with all 12 other frequencies. The Fourier phases are definitely between 0 and $\pi$, i.e., the oscillations should be coherent over all observational time spans (there is only less then half a period shift during 150000 periods).

If the $29.838 \mathrm{c} / \mathrm{d}$ frequency, with stationary amplitude, is added to the accepted set of solutions, a poorer overall fit to the data is obtained, and asymmetric cycles are created. This frequency was therefore omitted from the finally accepted solution.

A natural test of the quality of frequency solution is given by the fitting of the observed light curves. In Fig. 7 the fitted light curves are shown using the finally accepted frequency solution. Before we comment on the fit, we find it noteworthy that not only are there very low amplitudes of each excited mode (the amplitude ratio of the dominant modes for $\theta$ Tucanae and 57 Tau is 7.5 - Paparó et al. 1996) but there are complex light variations too. Specifically, these features are remarkable where only one cycle has "extremely" large amplitude both in minimum and maximum compared to the neighbouring cycles. Such dramatic changes in amplitude from one cycle to the other could hardly be explained by a missing frequency of a static pulsational arrangement.

In our conclusion the overall fit of the observed light curve is satisfactory in those parts where the stationary pulsational behaviour of 57 Tau dominates. The accepted frequency solution can yield the phase of minima and maxima in those parts. In some cases, the amplitudes of the synthetic light curves do not fit the observed one, where both lower or higher amplitudes occur. These cases suggest that more frequencies very close to the values used in the fit might be also excited. These frequencies could cause discrepancies only in the amplitudes not in phase. 


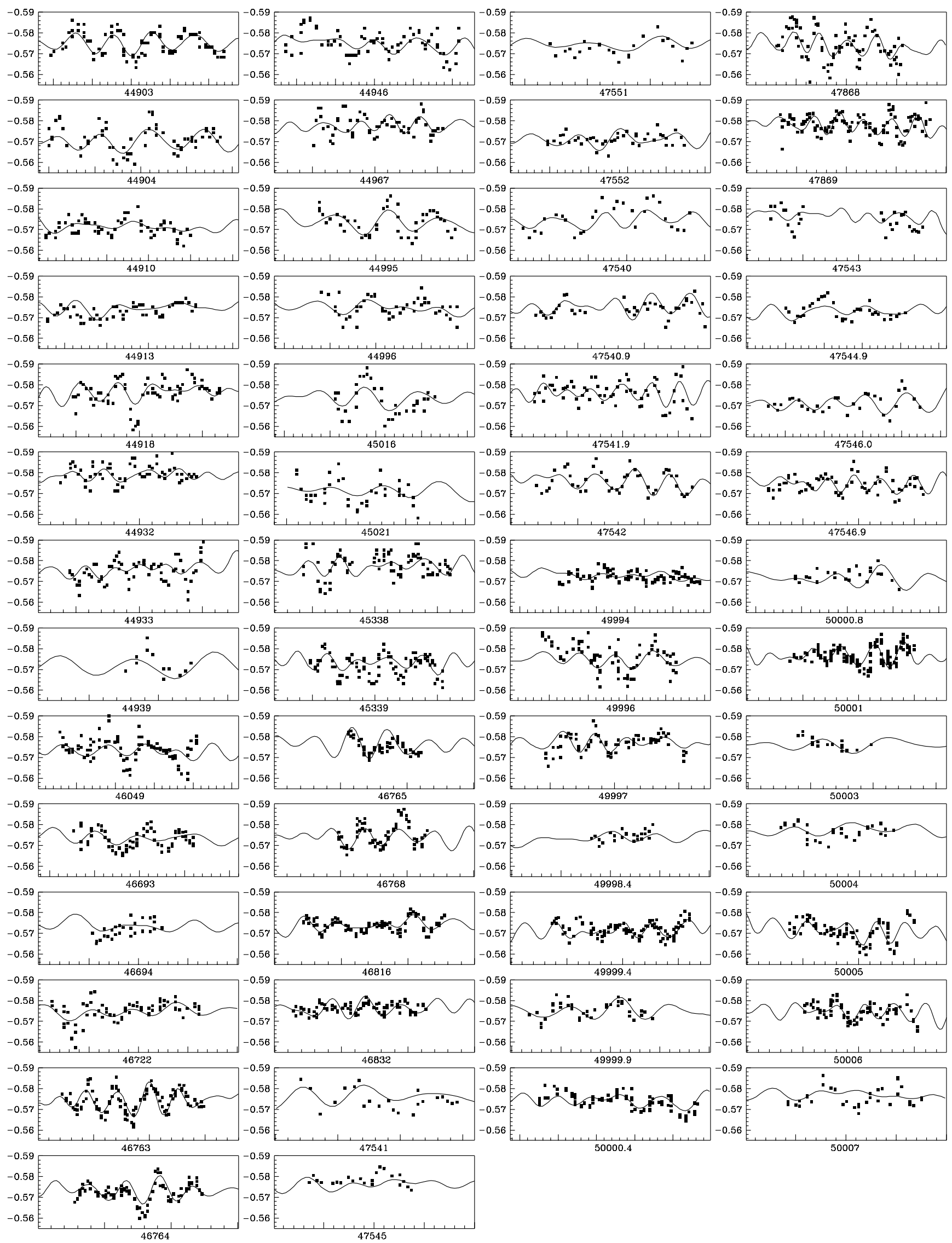

Fig. 7. Fit of the light curves for 57 Tau with the finally accepted frequencies from Table 5 . The date are given as HJD $2400000+$. The overall fit of the observed light curve is satisfactory in those parts where the stationary pulsational behaviour of 57 Tau dominates 

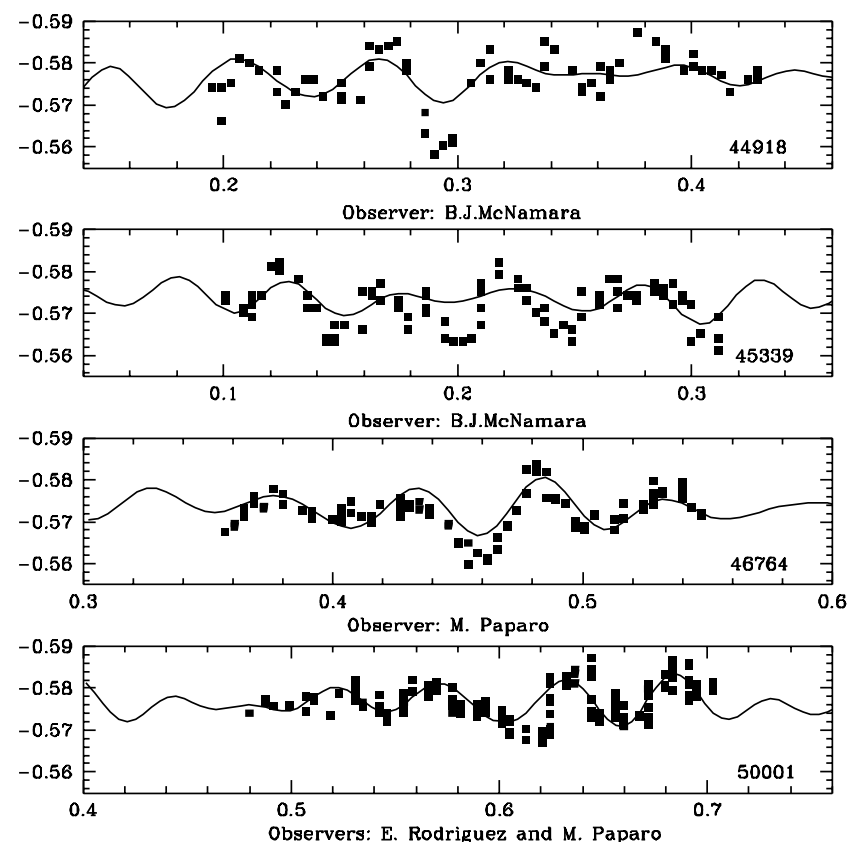

Fig. 8. Single unusual cycles of 57 Tau. The panels are marked as HJD 240000+. A physical process, gaining large enough observable size, from time to time, seems to be localized in the single unusual cycles

\subsection{Single unusual cycles}

The light variation of 57 Tau tends to resemble a slowly varying sinusoidal when $\mathrm{S} / \mathrm{N}$ is large enough. It is hard to say anything about nights when the amplitude of light variation does not exceed a $\mathrm{S} / \mathrm{N}$ of 1 . On some nights, however, single unusual cycles either with larger amplitude than the surrounding cycles or with asymmetric shape occur.

We focus the readers attention to the most remarkable cases, although similar events could be happening more often. In Fig. 8 those cycles are shown where the $\mathrm{S} / \mathrm{N}$ ratios are high and variations are large in amplitude or asymmetrical in shape. On night HJD 2444918 and 2446764 the single cycles have larger amplitude than the previous and consecutive cycles. On the other two nights, at HJD 2445339 and 2450001, the cycles look like a typical cycle of an RR Lyrae star, the ascending branch is very steep and the descending branch is less steep.

We should emphasize the following. As Fig. 8 shows the cited single unusual cycles were obtained by different (well-experienced) observers with different photometers on different sites. It is therefore extremely unlikely to be an instrumental effect. Each case was observed near to meridian, hence an explanation pertaining to the potential large-airmass differential observations is also ruled out. Although the amplitude of the general fit is exceptionally wrong for such cycles, they are still in phase with the fit. The previous and following cycles are well-fitted

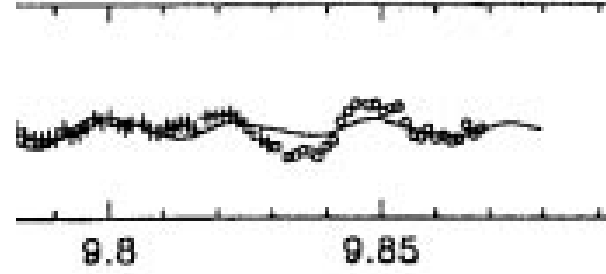

Fig. 9. Unusual cycle of XX Pyx observed in a WET campaign. Both before and after(!) the cycle, the amplitude is small and the fit is the same quality as it is near to the meridian for except the unusual single cycle

for these nights, and only the single cycles behave in an unusual manner. A physical process, gaining large enough observable size, from time to time, seems to be localized in the single unusual cycles.

A check for a possible connection between the frequency at $29.83 \mathrm{c} / \mathrm{d}$ and the unusual behaviour of the single cycles was carried out. Although the inclusion of this frequency marginally improves the fit for the single unusual cycles, it is hard to establish any definite connection because its effect is tiny.

It is well-known that a comprehensive study of a pulsating variable star, especially if the frequencies are not properly resolved, is not as convincing as, for example, a multi-site WET campaign. To support our finding of single unusual cycles in $57 \mathrm{Tau}$, the paper on CD - 24 7599 (=XX Pyx) observed in a WET campaign, published by Handler et al. (1996) is noteworthy. The data are excellent quality, the general fit is extremely good. However, the cycle at 9.85 in their Fig. 1. (see in Fig. 9 on larger scale) seems to be similar to what we see in 57 Tau. Although it is explained in the paper mentioned above as a consequence of the large airmass, both before and after(!) the cycle, the amplitude is small and the fit is the same quality as it is near to the meridian for except the unusual single cycle. Of course, such a tiny effect can be localized much better if it occurs more often in a star.

\section{Discussion}

The $\delta$ Scuti star, 57 Tau, seems to be unique in many aspects. The extremely small amplitude of the excited $p$-modes has been first mentioned by B.J. McNamara and is confirmed by the present study. The amplitude of the dominant mode excited in 57 Tau is only about twice as the residual amplitude level of $\theta$ Tucanae prewhitened with 13 frequencies (Paparó et al. 1996). We suggest two possible explanations for the periodicities in the low frequency domain. The more conventional, although nontrivial, explanation relates to the geometry of the system, i.e., a binary or multiple nature of 57 Tau. Spectroscopic observations of 57 Tau are urgently needed to check this explanation. The less conventional but more exciting explanation is that they represent the excitation of $g$ 
modes as well as the $p$ modes. According to its spectral type, 57 Tau is situated on HR diagram near to the cool border of the instability strip, exactly in the region where $\gamma$ Dor stars are located. The $g$-mode frequencies of $\gamma$ Dor stars discovered in the last few years (Abt et al. 1983; Antonello \& Mantegazza 1986; Krisciunas \& Guinan 1990; Balona et al. 1994; Breger et al. 1997; Zerbi et al. 1997; Kaye et al. 1998; Kaye et al. 1999a) occur in the same frequency range as in the low frequency domain of 57 Tau's amplitude spectrum. If this interpretation is correct 57 Tau would be the first $\delta$ Scuti type star where both $p \&$ high overtone $g$ modes are excited, i.e. where both $\delta$ Scuti-type and $\gamma$ Dor-type pulsation are found in the same star. This would present a great challenge to theoreticians. If the mass ratio were obtained from the binary (or multiple) system, and the chemical composition \& distance based on the membership of Hyades are assumed, 57 Tau is a good candidate for modelling. The possible excitation of the frequency at $29.83 \mathrm{c} / \mathrm{d}$ with variable amplitude and asymmetric shape of some single unusual cycles, might require non-linear and/or nonstationary processes.

High resolution, high SNR spectra of 57 Tau have been recently obtained by Kaye et al. (1999b). Subsequent analysis by Kaye (1999c) indicates that 57 Tau is a spectroscopic binary and some of the low frequency variations mentioned in the present paper "are probably due to duplicity (i.e. geometric and proximity) effects". Further multi-site observations of 57 Tau by the $\gamma$ Dor network are also recommended.

Acknowledgements. Hungarian research grants, OTKA Nos. T 021074 and T 025288, are acknowledged. MP acknowledges to the Hungarian Academy of Sciences and the Spanish Scientific Council (CSIC) for support of her visit to Spain. The hospitality of staff members in Granada (IAA) is greatly appreciated. ER and AR acknowledge to DGES for support under project PB 96-0840. The project was supported by the Chinese National Natural Science Foundation. The authors are indebted to Dr. E. Poretti for his referee report.

\section{References}

Abt H.A., Bollinger G., Burke E.W.Jr., 1983, ApJ 272, 196 Antonello E., Mantegazza L., 1986, AA 164, 40
Balona L., Hearnshaw J.B., Koen C., Collier A., Machi I., Mkhosi M., Steenberg C., 1994, MNRAS 267, 103

Breger M., Garrido R., Huang Lin, Jiang Shi-yang, Guo Zi-he, Frueh M., Paparó M., 1989, AA 214, 209

Breger M., Stich J., Garrido R., Martin B., et al., 1993, AA 271,482

Breger M., Beichbuchner F., 1996, AA 313, 851

Breger M., Handler G., Garrido R., et al., 1997, AA 324, 566

Eggen R.L., 1970, PASP 82, 274

Fu Jiang-ning, Jiang Shi-yang, Li Zhi-ping, 1996, A\&AS 115, 469

Handler G., Breger M., Sullivan D.J., van der Peet A.J., et al., 1996, AA 307, 529

Horan S., 1979, AJ 84, 1770

Kaye A.B., Henry G.W., Fekel F.C., Hall D.S., 1998, MNRAS (in press)

Kaye A.B., Handler G., Krisciunas K., Poretti E., Zerbi F.M., 1999a (PASP in press)

Kaye A.B., Guzik J.A., Neuforge C., Bradley P.A., 1999b, ApJ (in preparation)

Kaye A.B., 1999c, IBVS No. 4697

Kovács G., Paparó M., 1989, MNRAS 237, 201

Krolikowska M., 1992, AA 260, 183

Kolláth Z., 1990, Occasional Technical Notes, Konkoly Obs. 1

Kolláth Z., Paparó M., 1999 (in preparation)

Krisciunas K., Guinan E., 1990, Inf. Bull. Variable Stars No. 3690

Lampens P., 1987, AA 172, 173

Lopez de Coca P., Rolland A., Rodriguez E., Garrido R., 1990, A\&AS 83, 51

McNamara B.J., 1983, The Southwest regional Conference for Astronomy and Astrophysics, AA Abstract Publ., p. 43

Mermilliod J.-C., Mermilliod M., Hauck B., 1997, A\&AS 124, 349

Millis R.L., 1967, Ph.D. Thesis. Univ. Wisconsin

Paparó M., Sterken C., Spoon H.W.W., Birch P.V., 1996, AA 315,400

Paparó M., Saad S.M., Szeidl B., Kolláth Z., Abu Elazm, Sharaf M.A., 1998, AA 332, 102

Paparó M., Rodriguez E., Kolláth Z., 1999, in: Variable Stars as Important Astrophysical Tools, Ibanoglu C. (ed.), NATO ASI Conf. Ser. (in press)

Poretti E., Zerbi F.M., 1993, AA 268, 369

Rodriguez E., Lopez de Coca P., Rolland A., Garrido R., Costa V., 1994, A\&AS 106, 21

Zerbi F.M., Rodriguez E., Garrido R., et al., 1997, MNRAS 292,43 